\title{
From archive cultures to ephemeral content, and back: studying Instagram Stories with digital methods
}

\begin{abstract}
Despite growing interest, there is a shortage of research about the methods and challenges that concern researching ephemeral digital content. To fill this gap, the article discusses two research strategies to study Instagram Stories. These allow users to share moments of their everyday lives in a documentary and narrative style; their peculiar feature is ephemerality, as each Story lasts for 24 hours. The article a) explores how to by-pass the Instagram API closure and b) engages in an attempt at 'circumventing the object of study', taking advantage of how individual users archive Instagram Stories on other platforms (here, YouTube). In so doing, we contribute to the debate that seeks to innovate and 're-purpose' digital methods in a post-API environment. Besides the methodological utility, we show the tension between ephemeral content and archive cultures, and raise epistemological and ethical concerns about the collection, analysis and archival of ephemeral content.
\end{abstract}

Keywords: archive cultures, digital methods, ephemeral content, Instagram Stories, post-API

This article discusses two methodological strategies for the study of Instagram Stories. Released in August 2016, Instagram Stories is a feature of the social media Instagram that allows users to share "all the moments of your day, not just the ones you want to keep on your profile" (Instagram, 2016). Stories are characterized by a documentary, narrative and everyday style, and enable users to combine bring together the various modes of communication of allowed by the platform (pictures, videos, texts, emoji and stickers, audio) into a single digital object. Their peculiar feature is ephemerality, as each Story lasts for only 24 hours.

Despite growing interest, there is a shortage of research about the methods and challenges that concern researching ephemeral digital content. Existing literature so far has largely focused on providing a general understanding of ephemeral content as it appears on different platforms (Hagen, 2018; Koefed and Larson, 2016), chiefly on Snapchat, which where the MyStory feature is very similar to Instagram Stories. 
Scholars have mainly focused on how Snapchat's temporality mediates individuals' behaviours and practices by breaking the boundaryies between lived experience and documentation (Jurgenson, 2013), as well as on how it can shape memory, intimacy, and youth's sexual and relational cultures (Handyside and Ringrose, 2017; Koefed and Larson, 2016). In this context, the methodological strategies most often applied for the access, collection, analysis and archival of ephemeral content in the form of a 'story' rely on individuals' narrations collected through interviews and focus groups, often mixed with the observational analysis of online content. Yet, even when the focus is more centred on the content itself (Nashmi and Painter, 2018), current works largely avoid to-challenginge some key issues of accessibility, epistemology and ethics that the study of this new means of communication entails.

To fill this gap, the article experiments and critically discusses two possible research strategies to study Instagram Stories as an object of social and cultural research. In doing so, we intend to contribute to the emergent literature that seeks to innovate and 're-purpose' digital methods (Rogers, 2013) in a post-API environment (Perriam et al. 2019; Venturini and Rogers, 2019). On the one hand, we explore a way to by-pass the Instagram API closure, working around the restrictions to accessing Instagram data that have been enforced since April 2018'. On the other hand, we engage in an attempt at 'circumventing the object of study' (Bucher, 2017), taking advantage of the ways in which individual users archive Stories to ensure their permanence beyond ephemerality, and thus make them available on other platforms (in our case, YouTube). Besides the methodological utility, we also show the emergent tension between ephemeral and archive digital cultures; and discuss the epistemological and ethical questions that concern the modes of data collection, analysis and archival of ephemeral digital content. Our work is driven by two main questions: in which ways can digital methods be used for the analysis of Instagram Stories, and of ephemeral content more in-generally? Is the blending of qualitative and automated analysis a viable path for the study of Instagram Stories and ephemeral content?

\section{The rise of ephemeral content: Instagram Stories}


Despite the common understanding of the Internet as 'never forgetting' (MayerSchönberger, 2009), ephemerality has become a central component of many social media platforms, such as 4Chan (Hagen, 2018), Snapchat (Nashmi and Painter 2018), and Instagram (Vázquez-Herrero et al., 2019). All these provide affordances designed to allow the creation of content which disappears from users' view after a short period of time. Existing research has looked at the diffusion of ephemeral content focusing mostly on Snapchat. Attention has been paid not only to the content of ephemeral snaps (Nashmi and Painter, 2018; Roesner et al., 2014), but also to how the temporality afforded by the platform may influence and mediate users' subjectivities and interactions (Bayer et al., 2016; Utz et al., 2015), with a particular focus on the implications for self-presentation (Koefed and Larson, 2016) and intimate relationships (Handyside and Ringrose, 2017). Moreover, these studies highlight that the ephemerality that the the platform affords allows for a higher level of users' selfdisclosure (Bayer et al., 2016) and reduced self-presentational concerns as compared to the more edited and permanent content posted on Instagram (Koefed and Larson, 2016).

Despite the increasing interest in ephemeral content, however, scarce attention has been paid so far to Instagram and its Stories feature, characterized not only by ephemerality but also by multimodality and everydayness. Unlike Snapchat, Instagram has represented for a long time the preferred platform to share static and persistent images, allowing users to share snippets of everyday life (Hu et al. 2014), selfies (Senft and Baym, 2015), or to perform micro-celebrity practices (Marwick, 2015), with important implications for brands and marketers (Carah and Shaul, 2016). Since 2016, following changes to the platform's architecture the Stories format has become extremely popular, reaching the total amount of 500 million daily users in January 2019 (Newberry, 2019), and overtaking feeds as the primary way of sharing content (Constine, 2018). The rise of Instagram Stories reveals a tension between ephemeral content and archive cultures (Rogers, 2019) that is still overlooked in existing research. Handyside and Ringrose already questioned the simplistic definition of Snapchat as a disappearing social media, arguing that the platform offers "an intriguing mixture of stickiness and transience, perceived permanence and elusive ephemerality" (2017:12). This is also the case for Instagram, as a result of the platform's in-built affordances to archive Stories, namely the possibility to save 
ephemeral content in ad hoc folders called 'highlights', which are visible on each users' profile. Furthermore, external strategies and tools for scraping and downloading Instagram Stories (e.g. StorySaver) have emerged, together with cross-platform archival practices, particularly on YouTube. This suggests considering the creation, duplication and storage of Instagram Stories as an evolution of the forms of representation and archival of vernacular creativity (Burgess and Green, 2018) and a by-product of Instagram ranking cultures (Rieder et al., 2018). What's more, it is interesting to question how ephemerality blends with another constitutive element of Instagram Stories, namely its storytelling dimension. Thus, we build on the literature about stories and small stories on social media (Page, 2015; Georgakopoulou, 2017) to methodologically define our object of study.

\section{Exploring Instagram Stories: Methodological strategies}

From a methodological perspective, we build on the idea that Instagram Stories can be conceived as a kind of digital small stories (Page, 2015). These are a particular genre of online storytelling (conveyed through text, sound or image) through which a poster shares "mundane, ordinary and in some cases, trivial events" of her everyday life (Georgakopoulou, 2017: 268). Via digital small stories, Internet users display a particular representation of their selves, a cultural point of view or a moral vision, before a digital audience (Page, 2013). Different from traditional, offline small stories, digital ones configure as 'a-typical', that is, fragmented, open-ended and intertextual. Social media represent privileged sites for the proliferation of small stories, insofar as their architectures constantly invite users to express themselves by means of micronarrations (consider, for example, the Facebook's prompt 'what's on your mind?'). Instagram Stories follow the same logic, only more explicitly, since they qualify as ad hoc devices for everyday (micro)storytelling, that allow users to document mundane moments in their everyday life in real-time.

Besides the fleeting ordinary moments that they allow us to capture, Instagram Stories amount to be fleeting digital entities too. In fact, Instagram Stories last only a few seconds and, if not explicitly saved by the users on their own profile, they are no longer accessible after 24 hours. This ontological status makes these digital objects particularly challenging ts to study from a methodological point of view. That is why we hereby seek to devise ad hoc research strategies to locate, collect, analyse and 
archive ephemeral, story-format digital content such as Instagram Stories. The elusiveness of Instagram Stories not only lays in their content and format, but also in their technical status as data points. The Instagram APIs do not allow users to retrieve Instagram Stories. Thus, for example, the usually very useful Instagram Scraper (developed by the Digital Methods Initiative to collect Instagram posts by following hashtags and usernames, see Geboers, 2019) is not equally useful for exploring Stories. After the Cambridge Analytica scandal and the consequent curtailing of social media APIs (Bruns, 2019), Instagram Stories are among the social media data that cannot be (or can no longer be) obtained (Bruns, 2018). In the case of Instagram Stories, we can further speculate that this is due, on the one hand, to the private and personal information they contain or display, and on the other hand to the great business value they perceivably have (Puschmann 2019), since a lot of interaction among influencers and regular users takes place via Instagram Stories (Warren, 2019).

In order to address the methodological challenges that Instagram Stories raise, our strategies turn to follow the actors (Latour 2005) as we take advantage of the natively digital methods (Rogers 2013) Internet users use to capture and archive Instagram Stories themselves (Postill and Pink, 2012). In so doing, we seek the users' collaboration, making them, de facto, our co-researchers (Caliandro and Gandini 2017). Drawing on this epistemological principle, below we explore two methodological strategies, specifically consisting in: a) by-passing the Instagram APIs; b) circumventing the object of study.

\section{Strategy 1: by-passing the Instagram APIs}

Our first strategy consists in by-passing the Instagram APIs by using scraping techniques (Cooley et al., 1997). Scraping is an IT technique that enables researchers to grab specific digital entities (like the title of an article) directly from the HTML code of the web page in which they are located (Weltevrede, 2016). To put it extremely simply, scraping consists in programming ad hoc computer scripts to simulate the navigation of a human internet user who opens a given web page, and copy-andpastes its content onto another file (e.g. an Excel spreadsheet). Scraping is a 
controversial topic in academic research, of which the legal and ethical contours are fuzzy (Landers et al., 2016). Although not illegal per se (Waterman, 2020), scraping is a practice to which social media platforms are particularly averse, for a number of reasons. First, in order to pull the whole content of a web page, a script has to make a large number of 'calls' to the server hosting the web page. If this process is repeated multiple times, by multiple users, on multiple pages, it carries the concrete risk of engulfing the server and, eventually, crashing the website - a risk that social media companies want to ward off at all costs. Secondly, scraping permits to sneak into private profiles and access information that users are not necessarily willing to share. Last but not least, by using scraping techniques developers and researchers may access data - to some extent sensitive, such as lists of interests or the progression of likes over time (Rieder et al., 2015) - that social media companies do not intend to share, since their business model is based on the selling of such data (Zuboff, 2019). Yet, as argued by Venturini and Rogers (2019: 536-537), forms of scraping are a 'necessary evil' for social research if performed conscientiously. In their view, scraping "forces researchers to observe online dynamics through the same interfaces as the actors they study" (ibid.), and thus take advantage of the ways in which users themselves generate or manage content.

In our case, to scrape Instagram Stories we used a freely available tool: StorySaver². StorySaver allows you to visualize the Stories posted by users with public Instagram profiles, and to download them as .jpg or .mp4 files. The tool replicates the ephemerality of the object of study, as it allows for the collection of the Stories within the 24hours-frame of their permanence on each user's profile. We used the tool both as an interface to visualize Stories and a scraper to collect and organize them in an anonymized corpus.

\section{Ethical considerations about Strategy 1}

Although scraping is a controversial technique, we managed to employ it in a reasonably ethical manner. First, as mentioned, we collected data through one of the many free online tools for capturing public Instagram stories: StorySaver. Therefore, we used a scraping technique without programming our own scraping script in order to: a) bypass platform's restrictions or blocks (Chellapilla et al., 2005); b) disguise the non-human identity of the collector of data (Von Ahn et al., 2003); and c) access 
content protected with privacy settings or passwords (Franzke et al., 2019). In this way we have been compliant with the Terms of Service ${ }^{1}$ of Instagram (Fiesler et al., 2016). Second, we treated data in ways that caused no harm to users and were respectful of their privacy. We collected data by following 'neutral' stories (e.g. related to everyday errands) and avoiding those dealing with sensitive topics (e.g. political views, or sex). Consider also that, from 2018, users can activate the function 'close friends', which makes stories only visible to a list of chosen friends, hiding them from all other followers; this reduces the probability to run into content that users deem intimate. Given the difficulties often related to the request of consent in social media research (Salmons, 2015), we consider the data collected similarly to data gathered from observation methods (on this point see also Light et al. 2018 and their discussion on the 'walkthrough' method for the analysis of digital apps). Hence, we do not display any screenshots of Stories in this paper, nor share usernames, links to individual profiles or to any other personal information. Moreover, we analysed data in an aggregated as well as clustered form, and presented them through coding categories. Such categories are very general (such as 'portrait', 'landscape', 'mood', etc.) and, consequently, do not allow for the identification of users. Lastly, we did not share our dataset with third parties, since it was gathered exclusively for academic purposes. In short, we managed to address quite adequately the typical ethical issues that are normally ascribed to scraping techniques quite adequately, since we did not "[break] the law, or [put] a burden on a site's servers, or potential harm [...] users" (Fiesler et al., 2020: 10).

Although the procedures described above comply with general ethical and legal protocols, another matter of concern remains. Users are not always aware that their public data can be used for research purposes and might expect this to remain 'private' (Zimmer, 2010). Literature commonly concurs that this happens because, customarily, users do not read carefully the platforms' terms and conditions (Böhme and Köpsell, 2010) - also because they are often extremely long and difficult to comprehend (Reidenberg et al., 2015). Nonetheless, the fact that regular users tend to disregard platforms' terms and conditions cannot lead us to take for granted that they ignore, automatically, that social and corporate researchers might analyse web data. In fact,

\footnotetext{
${ }^{1}$ See https://www.instagram.com/about/legal/terms/api/.
} 
as Landers et al. have remarked, the recent privacy controversies (i.e. Cambridge Analytica) "have increased awareness that any data shared over the Internet has been in effect shared publicly" (2016: 487).

With reference to our specific case, we acknowledge that capturing Instagram Stories clashes with the conceptual notion of ephemeral content. It may be questioned whether researchers are actually legitimised in the first place to access, collect and archive for research purposes content that users produce in the understanding that it will soon become unavailable. It may be even pointed out that, compared to a traditional social media post, ephemeral content might be seen as a more privacyfriendly practice of posting by many users, as a way to publish materials that they do not want to remain permanently available on the platform. We contend this argumentation does not hold, for three reasons. First, while many users might post ephemeral content in the assumption it will be erased, this is not expressly guaranteed anywhere in the platforms' policies. Never, at any point, for instance Instagram explicitly claims that Stories will be deleted from the platform. Hence, we can't assume the opposite either, that is, that the average user expects her Stories to disappear completely. Consider the large number of 'story savers' available on Google Play and iTunes, which indicates the diffused users' practice to capture and archive each other's Stories. Second, as we will see in the section on YouTube, we have observed that users deem somewhat culturally valuable to duplicate certain ephemeral content - we as researchers followed the same principles.| Therefore, despite its ephemerality, we contend that as researchers we are obliged to treat ephemeral content just like any other content, and thus uphold to the same ethical standards and privacy prescription we take with all other types of digital content. Finally, it is worth making clear that we consciously adopted a data-activist stance. As argued by some of the most prominent digital scholars (Bruns, 2018; Kazansky et al., 2019), in an era of APIs curtailing social media researchers must engage in devising new and alternative methods to keep social media research alive; this with the ultimate scope to keep alive the critical thinking that most part of this research promotes (Bruns 2019).
Commentato [1]: Tagliato: Probably the diffusion of tracking apps brought about the Covid-19 emergency will accelerate this cultural process in the near feature.

Commentato [2]: Questo forse si può togliere da qui e risparmiare qualche parola, visto che lo dici già poco sopra?

Commentato [3]: si sono d'accordo, non mi dispiaceva ribadire la cosa, ma se siamo a corto di parole tagliamo pure ; )

Commentato [4]: Tagliato: Third, we treated personal data respectfully, since we didn't share our data with

third parties, we didn't publish personal data or display Stories, and we didn't display screenshots that could potentially harm our participants

Commentato [5]: Per quanto inspirational questa parte mi sembra però un po' superfula. cosa ne dite? se anche per voi non ci azzecca cancellate pure (o cosideratela come una parte sacrificabile in caso di word limit)

Commentato [6]: lo dico sacrificabile per word count Commentato [7]: ok, se non ci stiamo dentro con le parole tagliamo pure

Commentato [8]: Tagliato: Especially within digital research, ethical issues become inherently ambiguous, contextual-dependent and amenable to multiple responses (Fiesler et al. 2020). Anyway, this condition should not push researchers to stop doing research, discussing problems and looking for solutions 
Before starting our inquiry, we needed a point of entrance in the Instagram Stories ecosystem, in order to avoid collecting data in a too random and subjective way. Thus, we accessed the Instagram platform by following the generic hashtag \#happy. The choice of such a generic hashtag firstly allows us to run into users' ordinary content and, secondly, lowers the risk of incurring in sensitive topics that could have caused harm to users. Once set the keyword, we launched Instagram Scraper (DMI), which retrieved 10.000 posts containing the hashtag \#happy (March 2019 - April 2019). Then, we calculated the users' distribution per number of posts published. This operation permitted us to distinguish between very active users and less active ones. We concentrated our attention on the long tail and on that majority of users $(n=6.399$; $88,80 \%$ ) posting only once. We did so because, as mentioned earlier, we were interested in studying Stories posted by ordinary users - and, thus, seeking to avoid influencers and bots. In line with our operationalization of the notion of 'ordinary user', from this pool we randomly extracted a number of profiles accounting for two main requisites: the profiles a) are not bots, fan pages or brands, and b) have a number of followers lower than 2.000. Our final sample was composed by the first 15 randomly extracted users who matched these characteristics. Then, each user in the sample was followed for 7 days (25 April 2019 - 1 May 2019) and their stories collected every day of the week at the same time, in order to account for the daily Stories-sharing activities. Through this procedure we created a corpus of 292 Stories. The resulting empirical materials were then organized in a spreadsheet containing an arbitrary id label for each Story, the date of extraction, an anonymized user id, together with the transcription of the texts and audio content of each Story. The data collection procedure was also accompanied by ethnographic notes taken by the authors while first observing the Stories.

\section{Data analysis}

At the time of writing, the scraping procedure using StorySaver only allows to collect Stories from a single user. This peculiarity, together with the ethnographic and manual work required to gain the empirical material, does not allow for the creation of an extended dataset. Given these characteristics we analysed Instagram Stories' visual and audio content by means of an ethnographic coding approach (Altheide, 1987), 
that blends ethnographic and non-intrusive participant observation and note-taking with coding practices from the content and visual analysis traditions. More specifically, the analysis builds on the integration of compositional and content analysis as intended by Rose (2016), with a specific attention for both still and moving images (Heat et al., 2010). Given the exploratory nature of the study, and in line with the principles of an ethnographic coding approach (Altheide, 1987), the existing literature on Instagram (e.g. Leaver et al. 2020; Manovich, 2016) initially guided the creation of the visual codes, while other descriptive and analytical labels were expected and allowed to emerge throughout the study.

Moving from the denotative to the connotative level (Banks, 2007), the analysis aims at grasping the visual content and practices of use of Instagram Stories, and consists in five steps, summarised as follows:

Denotative level:

1. Format

2. Visual codes

Connotative level:

3. Narrative style

4. Context of use

5. Grammars

We started the analysis by focusing on the denotative level and visual content of the Stories collected, taking each Story as a single unit of analysis (Rose, 2016). In order to account for both still and moving images, we started analysing the 'format' of each story, distinguishing between static pictures (66\%) and videos and small animations (34\%). Given the short duration of Instagram Stories (max 15 seconds), we noticed that the videos we collected can be considered as the transposition of a singular event in a dynamic format. For this reason, we followed a similar procedure for the visual content analysis of both static and dynamic content, focusing on the main elements represented. 
Secondly, we delved into the content of each Story accounting for the predominantly visual codes. In particular, we looked at two types of codes: a) content-related codes, addressing what is represented in each photo/video; and b) Instagram-specific digital objects, meaning the visual elements inscribed within the platform affordances that allow to create and beautify Instagram Stories - specifically stickers (e.g. emoji, gif) and what we call 'interactive stickers' (e.g. poll; slide emoji stickers ${ }^{3}$ ). We then analysed and coded all the Stories in the dataset, describing the predominant visual elements and at the same time looking for patterns, similarities and differences within the corpus. After open-coding each video and photo, we grouped the denotative labels into broader visual content categories (Figure 1).

\section{Figure 1 - Visual content analysis - Coding categories}

Insert Figure 1

As Figure 1 shows, the most recurrent visual components represented in our corpus refer to the category 'portrait' (33,90\%), mostly showing users themselves (labelled as 'selfie', $20 \%)$, or alternatively friends (7\%) and family (3\%). Instagram Stories are also displaying material assemblages (indicated with the category 'materiality', 12,35\%), characterized by the presence of both material objects $(6 \%)$ and body parts $(4 \%)$. Other recurrent visual elements are the representation of specific settings and landscapes $(10,95 \%)$, celebrations $(9,93 \%)$ and food $(9,24 \%)$. Both the categories 'portraits' and 'materiality', two of the most recurrent ones, reflect the most common visual elements represented in Instagram posts (Hu et al., 2014), pointing to the persistence of some visual codes considered Insta-worthy of being shared in spite of their ephemerality. The ways in which these objects are photographed and combined together recalls the presence of specific Instagram aesthetics, such as the recurrent ways of presenting brands as assemblages (Rokka and Canniford, 2016) and conventions in representing selfies (Hess, 2015).

Besides these predominant visual elements, the second most recurrent visual category found is the one coded as 'composition' (22\%). The Stories within this 
category are characterized by the juxtaposition of different elements, mostly text $(10 \%)$, stock images $(5 \%)$ and memes $(2 \%)$. Notably, in this type of Stories the main focus is not on a visual element, but rather a composition of texts and images created by the user. In this case, the denotative level of the pictures seems to take a back seat, as it is the juxtaposition of different elements which orients the focus and meaning of the Story. Moreover, looking at the distribution of digital objects, it can be noted that, despite the ubiquitous presence of GIFs and emojis in almost all the Stories collected, 'interactive stickers' become an essential element in the composition category (3\%). Digital elements inscribed within the platform's affordances are thus used in concert with more traditional visual elements, creating a composition that is peculiar to the Stories-format. It is in this multimodality that lies one of the main specificities of Instagram Stories against the 'classic', more static, Instagram posts.

In the second part of the analysis, we moved to the connotative level and examined the Instagram Stories in relation to their broader cultural meanings. First, we considered the 'narrative style' of the Stories in our corpus. In line with the definition of 'small stories' and their peculiarities previously outlined, the results show that the majority of the empirical material analysed is composed by Stories as single units $(60 \%)$, namely, single snippets with their own meaning. The remaining $40 \%$ is organized in coherent narrations, which assume the form of a documentation of a given event by means of different frames juxtaposed in a slideshow, rather than the articulation of a linear story. Despite the presence of some micro-narrations in a very documentary style, each Story maintains a meaning on its own, and its content as well as its contextual use can easily be understood without taking into consideration the whole narration in which it is set.

Therefore, moving to the interpretation of Instagram Stories' 'context of use' we analysed each video and image in itself, while taking into account the other Stories shared by the same user as contextual elements. This procedure resulted in the creation of 7 categories representing different ways to interpret Instagram Stories as a social practice (Figure 2).

Figure 2 - Instagram Stories - Context of use 
Insert Figure 2

Not surprisingly, the vast majority of Stories accounts for what we labelled 'special events' $(37,33 \%)$, namely those moments considered by users as special, out of the ordinary, and thus worthy of being recorded and shared. The second most popular category includes snaps representing moments of everyday life (25,68\%). Moreover, and in line with the presence of portraits and selfies, a certain number of stories is aimed to share an image of oneself ('self-display', 7,88\%). Two other categories are worthy of attention, what we called 'mood' $(10,62 \%$ - Stories expressing personal feelings) and 'interaction' $(9,59 \%$ - Stories that attempt to establish a flow of communication with followers).

Lastly, matching the visual codes of the analysis (Figure 1) with the context of use (Figure 2), we were able to identify some specific 'grammars' (Figure 3). The concept of 'grammar' points to a set of aesthetic norms characterizing Instagram Stories, as well as the possibilities offered from the platform to users' actions (Gerlitz and Rieder, 2018). In this sense, the idea of grammar takes into consideration the visual elements and how they are combined in their compositional modality (Rose, 2016), the cultural meaning they reflect, and the role of platforms in prompting them.

Figure 3 - Visual categories distributed per context of use

Insert Figure 3

Figure 3 shows the different types of visual elements associated with specific Instagram Stories' context of use (just the four most recurrent ones are represented). It emerges that the narration of special events is mostly represented by means of portraits (44,95\%) and celebrative moments (25,96\%), whereas the 'daily life' context of use is characterized by the presence of material assemblages (32\%) and the representation of everyday settings (24\%). Yet, we can see that users communicate their mood and try to create interaction mostly by means of the 'composition' type of 
Stories (74,1\% and $60,71 \%$ respectively), thus relying on the juxtaposition of different visual and textual elements. According to these results, two main grammars can be outlined: a grammar for documentation and a grammar for interaction. The first one is related to the celebration of special events as well as to the representation of ordinary, everyday moments, and it is based on portraits and materialities. These results support the idea that Instagram Stories promote the visual publicization of ordinary life in a context characterized by ubiquitous photography (Hand, 2012). With their Stories, regular users seem to reaffirm the original purpose of Instagram as a social media for sharing pictures taken on-the-go which has recently been challenged by the presence of staged and polished content, mostly posted by celebrities and influencers.

The second grammar, called 'grammar for interaction', is mostly characterised by the 'composition' type of Stories, which are used to share one's mood and to generate interaction. This grammar seems particularly influenced by the indications of how-to tutorials suggesting the best way to create engaging content, visibility and revenues (more on this later). In particular, with the 'compositional' type of Stories users tend to reflect the practices adopted by celebrities and influencers in a well-known form of micro-celebrity transposed to the Stories-format (Marwick, 2015). Despite the adoption of codes and practices related to marketing-related content, however, these attentionseeking practices seem less directly aimed to outsmart the Instagram algorithm but, rather, recall forms of what Bucher (2018) calls 'programmed sociality'. Such a concept is useful to explain how communication flows and interactions through Instagram Stories are oriented by the platform's affordances and at the same time measured and evaluated according to the ubiquitous criterion of engagement. In this sense, the grammar for interaction can be considered as one of the means by which the platform's affordances function to encourage users to connect and engage with each other.

The analysis has shown the visual peculiarities and the contextual use characterising Instagram Stories. The two resulting grammars allow to highlight how the multimodality typical of the Stories feature blends with a documentary style and with the possibility to generate flows of communication through the Stories. These elements, together with the ephemerality of the medium, represent the peculiarities of Instagram Stories compared to the traditional Instagram feed. Focusing instead on the aesthetics of the visual texts in our corpus, despite the presence of Stories-specific grammars, some 
compositional norms related to the broader platform vernacular (Gibbs et al., 2015) still persist. Not only traditional Instagram-worthy elements are represented, such as selfies and assemblages of material objects; also the ways in which they are depicted and framed are in line with already-established Instagram aesthetics (Manovich, 2016). Thus, despite the feature of spontaneity embedded in the Stories' ephemerality, the moments captured continue revealing some platform-specific compositional norms. The ephemerality of Instagram Stories thus allows users to share more spontaneous content, which is nonetheless somehow coherent with broader Instagram aesthetics.

\section{Strategy 2: circumventing the Instagram platform}

An alternative strategy for the study of Instagram Stories is the possibility to 'circumvent the object of study'. Due to its peculiar nature of content that is destined to disappear, we assumed that ephemeral digital content was prone to practices of duplication, storage and archival by users who would want to preserve it because they consider it somewhat culturally valuable or simply keep it available to others beyond its 'expiry date'. Accordingly, we expected to find Instagram Stories replicated and stored outside the Instagram platforms, particularly where audiovisual 'vernacular' content is already commonly archived and made available to others for sharing and commenting. For these reasons, we turned our attention to YouTube, that is widely considered to be the digital repository of present-day popular culture (Burgess and Green, 2018).

The strategy of 'circumventing the object of study' is not new in digital social research. Here, we take inspiration from Gerrard (2018), who located pro-anorexia communities on Instagram, Pinterest and Tumblr by 'circumventing' hashtags (such as \#proana or \#thinspiration) as a mechanism of search (since members of those communities explicitly avoid using hashtags in their messages), and particularly from Bucher (2017), who was interested in studying an invisible (but crucial) digital entity: the Facebook algorithm. Instead of considering it a 'black box' and hence give up her inquiry, Bucher decided to explore the tweets in which users reflected on how the Facebook algorithm works. Although they were not accurate accounts from a technical 
point of view, such mundane reflections actually allowed Bucher to grasp a key component of the Facebook algorithm: the social imaginary that ordinary users coconstruct around it (Bucher, 2017). In our case, we circumvented the Instagram platform by searching for Instagram Stories on YouTube. This strategy is pointed at accessing Stories that users duplicate and store in the implicit assumption of their relevance. In this sense here we follow the actors in a way that is practically different, but epistemologically analogous to Strategy 1 .

The use of YouTube to 'circumvent the object of study' and thus search for Instagram Stories outside the Instagram platform has several advantages. First, it allows to observe Stories that do not expire after a set time - thus eluding the ephemeral nature of this kind of digital content and the ensuing time-related difficulty that its analysis might entail. Hence, it allows to collect a high number of Stories at once and store them in a dedicated database. Secondly, from an ethical perspective YouTube presents far less issues of access, since it does not require to by-pass the restrictions that pertain to the Instagram API. The YouTube platform is arguably more open to forms of digital methods, and its practices of use in digital social research are wellestablished and commonly accepted (see Rieder, 2015; Rieder et al., 2018). Third, YouTube provides researchers with metadata that are crucial to understand the social impact of Stories but are not otherwise provided by the Instagram platform - in particular, reactions (e.g. likes, comments) to each (replicated) Story. Yet, as we are about to see, YouTube also has the evident disadvantage of gathering Stories that, for the most part, are not by ordinary users. On the contrary, most of the content tagged as 'Instagram Stories' on YouTube consists of videos featuring celebrities and/or influencers, or tutorials on how to professionally produce Instagram Stories. Nonetheless, although not posted by ordinary users, YouTube allows to understand which kind of Stories ordinary users deemed relevant and worthy of being archived, and to follow them as digital objects in a more aggregated manner, as opposed to Instagram which at present allows for a more user-centred approach.

\section{Data collection}

Using the Video List module that is part of the YouTube Data Tools (Rieder, 2015), we performed a data collection starting from the keyword 'Instagram Stories', deployed in May 2019 (5 iterations). This enabled us to obtain a dataset of 11669 videos related 
to this initial query. At first inspection, these include a variety of materials, not only Instagram Stories, such as music videoclips, excerpts of TV programmes, and much more. This is indicative of how the 'Instagram Stories' tag is used as a popular marketing hook to make sure content is found on YouTube.

To browse this variegated dataset, we extrapolated only those items where the occurrence 'stories' was present in the video label and/or channel title. This rendered a dataset of 1732 videos where the occurrence 'stories' appears in the video title, and 562 videos where this entry appears in the channel title. Only 365 videos appear in both datasets.

Figure 4 - Video Title occurrences, 'stories', per Category

Insert Figure 4

Figure 5 - Video Title occurrences, 'stories', ordered per view count, top 10

Insert Figure 5

This reveals a prominence of content from the People and Blogs category (Figure 4), that divides essentially in two categories: a) how-to-content and marketing related videos (Figure 5) and b) replicated content by celebrities and/or influencers - in particular, in our case, of pop icon Chiara Ferragni (Figures 6 and 7).

Figure 6 - Channel title occurrences, 'stories', top 10

Insert Figure 6

Figure 7 - Videos of Channels that include 'stories', ordered per view count, top 10 Insert Figure 7 
Alongside performing qualitative content analysis (Altheide, 1987) to inductively analyse the content of these videos, as said the use of YouTube provides the unique advantage of analysing metadata that are not otherwise retrievable via the Instagram platform, which may be equally (if not more) illustrative of the meaning, uses and circulation of Instagram Stories. For instance, we can analyse the comments under the replicated Stories, and thus observe the reactions of users to the content they watch. This is particularly interesting insofar as reactions to Instagram Stories are not publicly visible on Instagram, but are only accessible to the creator of the Story.

As an example, again using the YouTube Data Tools we retrieved the comments pertaining to the most viewed video in our sample of replicated Instagram Stories, entitled "La nascita di Leo" (English translation: "The birth of Leo"). This is a collection of Instagram Stories by Fedez (the husband of Chiara Ferragni) that depict the birth of their son Leo. The video has 901 comments, the vast majority of them in Italian language. These comments can be analysed in various ways. In this example, first we performed an exploratory qualitative analysis of the comments ordered on the basis of the 'reply count' feature. This allows to highlight 'controversial' comments that spark more conversation among users, and thus rapidly take a glance at the nature of the ensuing debate. The analysis suggests the presence of a highly polarised conversation around the practice of documenting everyday life by celebrities using Instagram Stories. Comments with more replies seem to largely split in two types: on the one hand, we have a group of positive reactions, which sees many users congratulate the couple for their newborn child. On the other hand, we can see a lively discussion taking place around the documentation of the child's birth using Instagram Stories made by the couple. To corroborate this insight, we then performed a sentiment analysis of the comments, which have been manually coded using: $1=$ positive comment; 2 = negative comment; 3 = neutral/unrelated comments. Overall, the analysis confirms the divided data and the related polarization. The relative majority of the sample is made of positive reactions (44,93\%); while there is a relevant presence of unrelated or neutral content $(31,01 \%)$, there is also a considerable presence also of negative comments $(24,06 \%)$. 
Figure 8 - Topic analysis, comments to "La nascita di Leo" (percentage values, filtered per sentiment)

Insert Figure 8

Alongside the sentiment analysis, we also performed a topic analysis by manually coding each of these comments on the basis of the topic it deals with. If we filter the topic analysis per sentiment (Figure 8), we can see the narrations that accompany this conversation, which split between positive positive comments about the couple (here tagged as 'celebrity' content) and the baby (tagged as 'baby'), and negative comments about the use of Instagram Stories made by the couple to document their child's birth (here tagged as 'documentation'). These criticize the way the couple rendered the birth of their son a piece of showbiz, such as the example below:

"yeah but it's not normal that you are giving birth and you only think about filming it" 5

Others, however, defend the choice of the couple to document the birth of their child on Instagram Stories:

"I find this anger totally out of place. "They are the only ones who had a child..." If you're annoyed by the fact they publicly disclose something, you should not be here commenting and giving views, it's called coherence. I never liked Fedez (Ferragni's husband, ndr) as an artist but I must say that in these pictures I've seen a touching dad, and believe me he is the first one to laugh reading the silly things you write."6

This kind of analysis, we argue, is quite insightful for two main reasons. On the one hand, we can clearly evidence how Instagram Stories are clearly understood as culturally contentious objects by users and represent a key marketing tool beyond the boundaries of the Instagram platform. On the other hand, we have been able to observe and decipher ephemeral digital content as a cultural object in a way that the Instagram platform does not allow (irrespective of its API closure), enabling us to expand our understanding of Instagram Stories in their cultural complexity.|
Commentato [9]: ELIMINATO:

"it looks like they are the only ones who had a child.. what a bummer" 4

This kind of comment can be observed many times throughout the entire thread, often accompanied by a pointed critique on the appropriateness of documenting a child's birth on Instagram Stories. An example is the comment below:
Commentato [10]: Anche qui ho tolto qualcosa e provato a sintetizzare. Ho rimosso la reference a Marres in fondo alla sezione perchè oltre a essere sbagliata $\mathrm{mi}$ sembrava ridondante (e ci salva qualche parola). Leggete se vi torna comunque. 


\section{Final discussion, limitations and further research}

The article has experimented two research strategies to 'repurpose' digital methods for the study of ephemeral digital content such as Instagram Stories, which represents an emergent, relevant and understudied dimension of present-day digital cultures.

On the one hand, we suggested the use of scraping techniques as a means to access and observe ephemeral content in the timeframe it is made available by users. As such, scraping techniques empower the researcher to organize ephemeral content for cultural analysis and delve deep in its multi-modality, thus offering a rich picture of ephemeral content as a social and cultural entity. Yet, while offering insights on the content of Instagram Stories, the use of scraping techniques does not exhaustively address ephemeral content as a 'flow' that cannot be extricated out irrespective of its context, an aspect which, depending on the research question, may be to consider in the research design.

On the other hand, by circumventing the object of study we were able to observe how, despite the rise of ephemeral content, digital cultures remain prominent milieus for archival practices of vernacular creativity (Burgess and Green, 2018), particularly of content that is designed to disappear. The study of the duplication of Instagram Stories on YouTube enables researchers to investigate more closely the context within which ephemeral content appears, its function and perception as offered by the comments, which represent extremely valuable pieces of information otherwise unavailable on the Instagram platform.

Despite their experimentation in isolation, we contend it is the combination of these strategies that offers a rich set of insights. First, by using digital methods instead of traditional observational techniques we were able to capture and store Instagram Stories in dedicated databases, which allowed us to perform more refined qualitative analysis. Moreover, the combination of the two strategies granted us to investigate and cast a light on the underlying mechanisms of influence between platforms and users, which were not possible to unearth otherwise. Specifically we saw that, notwithstanding Instagram Stories encourage ordinary users to express their own creativity - a call that users genuinely embrace - they tend to do that by adhering to
Commentato [12]: In rosso: ho ampliato la sezione di discussione con dei frammenti già scritti in una delle precedenti versioni del paper. Valutiamo insieme che cosa tenere, togliere e che cosa aggiungere!

Commentato [13]: questa parte mi piace molto. cmq se volgiamo recuperare spazio si può togliere la parte in cui si far riferimento a Nieborg and Poell

Commentato [14]: Tagliato e modificato: Yet, while it offers insights on the content of Instagram Stories, the use of scraping techniques does not exhaustively address ephemeral content as a 'flow' that cannot be extricated out irrespective of its context. As Nieborg and Poell point out, "cultural commodities become

fundamentally "contingent," that is increasingly modular

in design and continuously reworked and repackaged,

informed by datafied user feedback" (2018: 4275).

Accordingly, it may be said that by using scraping the

'flow' of ephemeral content gets partly lost in the

process of analysis, which, depending on the research

question, may be an aspect to consider in the research design.
Commentato [15]: Qui rileggendo forse si può esplicitare "observational" invece di "qualitative"?

Commentato [16]: Si io sono d'accordo! 
the Instagram platform aesthetics as well as norms of influencer marketing. Finally, our study shows how ephemeral and archival cultures coexist across social media platforms in a seamless dimension. The study of ephemeral and archival cultures as separate and neatly distinguished entities does not allow to grasp the extent to which ephemeral and traditional content actually interact and complement each other. Our analysis of Instagram Stories, albeit primarily pointed at methodological reflections, ultimately shows that ephemeral content adds a new layer of complexity to digital cultures and its peculiar practices, insofar as it does not suppress the cultural logics of archival, but embeds within it, in a blending of new and established practices and forms of sociality...

Finally, it is worth acknowledging that, however useful, our methodology is not privy of limitations, which relate to both our global methodological framework and the two specific strategies.

Regarding the general framework, we acknowledge the limited number of cases our empirical research draws on. This was due to the exploratory nature of the project. We hope that thanks to our protocol of analysis further research will be able to address a higher number of cases. Furthermore, we focus only on two social media platforms; it is our conviction that a cross-platform approach (Rogers, 2019) (for instance considering also Twitter, Tumblr, Pinterest or TikTok), could deepen our understanding of Instagram Stories as technical and cultural devices as well as of the tension between ephemeral and archive cultures. Concerning Strategy 1, for how systematic and transparent, we acknowledge that our procedure to identify ordinary users and content on Instagram might sound somewhat arbitrary. As our research project is the first of this kind, we needed an initial point of entry into the Instagram Stories ecosystem. Yet, further big data and statistical analysis could help in devising more objective data collection processes, for example, by: a) identifying ad hoc hashtags and/or public Instagram pages that primarily aggregate ordinary Stories; $b$ ) automatizing the procedure to detect bots, fan pages and influencers; c) estimating the average number of followers of ordinary users. Strategy 2, in turn, presents two main limits. First, collecting Stories through YouTube means primarily running into celebrity or influencers' Stories - although not directly posted by these actors. Second, while YouTube allows researchers to access the public debate around Instagram
Commentato [17]: secondo me se siamo a corto di parole questo si può sacrificare, che ne dite? Commentato [18]: Si sono d'accordo!

Commentato [19]: Occhio che "protocol" è parola connotata, la cambierei con altro (ad es "thanks to these experimentations")

Commentato [20]: qui si potrebbe menzionare i profil aggregatori di storie del Covid (\#stayathome)

Commentato [21]: Ho provato ad aggiungere in nota questa parte, ma si può spostare (o eliminare per il word count)

Commentato [22]: molto fico. cmq concordo che se siamo a corto di parole questa parte si può eliminare. NOTA (che non richiede risposta ora). E se nel futuro prossimo pensassimo ad un articolo quick and dirt in cu usiamo questa metodologia per studiare la cultura del covid? che in effetti è attraversata da una tensione tra l'oggi ed il futuro che verrà (magari lo facciamo in italiano)

Commentato [23]: Tagliato:

For example, in an ongoing research about the forms of mediated sociality during the Covid-19 pandemic, we used public Instagram pages re-post regular users' Stories with the hashtag \#iorestoacasa (\#stayathome) as in-platform aggregators of Stories for data collection and analysis. 
Stories, yet it is not possible to be sure that those reacting to and commenting Storiesrelated videos are also users that actually and routinely consume Instagram Stories or have a particular interest in them. Again, big data and statistical analysis can be helpful here. For example, a large-scale mapping of YouTube - perhaps also using the YouTube Data Tools - can help researchers to detect specific communities of Instagram users posting and discussing ordinary Stories.

\section{Notes}

1 See https://www.instagram.com/developer/changelog/ (Last accessed 16 November 2019).

2 See https://www.storysaver.net/ (Last accessed 10 December 2019).

3 See https://help.instagram.com/151273688993748 (Last accessed 10 December 2019).

${ }^{4}$ Original comment in Italian (translated by the authors).

${ }^{5}$ Original comment in Italian (translated by the authors).

\section{References}

Altheide DL (1987) Reflections: Ethnographic content analysis. Qualitative sociology 10(1): 65-77.

Banks M (2007) Using visual data in qualitative research. London, UK: Sage.

Bayer JB, Ellison NB, Schoenebeck SY and Falk EB (2016) Sharing the small moments: ephemeral social interaction on Snapchat. Information, Communication \& Society 19(7): 956-977.

Böhme R and Köpsell S (2010) Trained to accept? A field experiment on consent dialogs. In Proceedings of the SIGCHI conference on human factors in computing systems, 2403-2406.
Commentato [24]: Ho editato appena la parte su YT per evitare ripetizioni con la mia ggiuntaa sopra

Commentato [25]: ottimo 
Bruns A (2018) Facebook shuts the gate after the horse has bolted, and hurts real research in the process. Internet Policy Review. Available at: https://policyreview.info/articles/news/facebookshuts-gate-after-horse-hasbolted-and-hurts-real-research-process/786 (accessed 8 February 2020).

Bruns A (2019) After the 'APIcalypse': social media platforms and their fight against critical scholarly research. Information, Communication \& Society 22(11): 1544-1566.

Bucher T (2017) The algorithmic imaginary: exploring the ordinary affects of Facebook algorithms. Information, communication \& society 20(1): 30-44.

Bucher T (2018) If...Then: Algorithmic Power and Politics. Oxford University Press.

Burgess J and Green J (2018) YouTube: Online video and participatory culture. Hoboken, US-NJ: John Wiley \& Sons.

Caliandro A and Gandini A (2017). Qualitative research in digital environments: A research toolkit. London: Routledge.

Carah N. and Shaul M (2015) Brands and Instagram: Point, tap, swipe, glance. Mobile Media and Communication 4(1): 69-84.

Chellapilla K, Larson K, Simard PY and Czerwinski M (2005) Computers beat humans at single character recognition in reading based Human Interaction Proofs (HIPs). In CEAS. Available at https://www.semanticscholar.org/paper/Computers-beat-Humans-at-Single$\underline{\text { Character-in-based-Chellapilla- }}$ Larson/e405e0f1bca55b7d0e17656533f63fd025c2dd58 (Accessed 29 April 2020). 
Constine J (2018) Stories are about to surpass feed sharing. Now what? Techcrunch. Available at https://techcrunch.com/2018/05/02/stories-are-aboutto-surpass-feed-sharing-now-what/ (Accessed 10 February 2020).

Cooley R, Mobasher B and Srivastava J (1997) Web mining: Information and pattern discovery on the World Wide Web. In Proceedings of the Ninth IEEE International Conference on Tools With Artificial Intelligence: 558-567.

Fiesler C, Lampe C and Bruckman AS (2016) Reality and Perception of Copyright Terms of Service for Online Content Creation. In Proc. ACM CSCW 2016, $1450-1461$

Fiesler C, Beard N and Keegan BC (2020). No Robots, Spiders, or Scrapers: Legal and Ethical Regulation of Data Collection Methods in Social Media Terms of $\begin{array}{llll}\text { Service. } & \text { cmci.colorado.edu. } & \text { Available }\end{array}$ https://cmci.colorado.edu/ cafi5706/ICWSM2020 datascraping.pdf (Accessed 29 April 2020).

Franzke AS, Bechmann A, Zimmer M and Ess C. (2019) Internet research: Ethical guidelines 3.0: Association of internet researchers. Available online: https://aoir.org/reports/ethics3.pdf.

Georgakopoulou A (2017) 17 Small Stories Research: A Narrative Paradigm for the Analysis of Social Media. In: Sloan L and Quan-Haase A (eds.) The SAGE Handbook of Social Media Research Methods. London, UK: SAGE, pp. 266281.

Gerlitz C and Rieder B (2018). Tweets are not created equal: Investigating Twitter's client ecosystem. International journal of communication 12: 528-547.

Gerrard Y (2018). Beyond the hashtag: Circumventing content moderation on social media. New Media \& Society 20(12): 4492-4511. 
Hagen S (2018) Rendering legible the ephemerality of 4chan/pol/. Oilab. Available at https://oilab.eu/rendering-legible-the-ephemerality-of-4chanpol/ (Accessed 10 February 2020).

Hand M. (2012). Ubiquitous photography. Cambridge: Polity.

Handyside S and Ringrose J (2017) Snapchat memory and youth digital sexual cultures: mediated temporality, duration and affect. Journal of Gender Studies 26(3): 347-360.

Heath C, Hindmarsh J and Luff P. (2010). Video in qualitative research. London: SAGE.

Hess A (2015) The selfie assemblage. International journal of communication 9(Feature): 1629-1646.

Hu Y, Manikonda L and Kambhampati S (2014) What we instagram: A first analysis of instagram photo content and user types. Eighth International AAAI conference on weblogs and social media.

Instagram (2016) Introducing Instagram stories. Available at: http://instagram.tumblr.com/post/148348940287/160802-stories (Accessed 10 February 2020).

Jurgenson, N. (2013). Pics and it didn't happen. The New Inquiry. Available at: http://thenewinquiry.com/essays/pics-and-it-didnt-happen/ (Accessed 10 February 2020).

Koefed J and Larson MC (2016) A snap of intimacy: Photo-sharing practices among young people on social media. First Monday 21(11): 1-9. 
Kazansky B, Torres G, van der Velden L, Wissenbach K and Milan S (2019) Data for the social good: Toward a data-activist research agenda. In: Daly A, Devitt SK and Mann M (Eds.) Good data. Amsterdam: Institute of Network Cultures, pp. 244-259.

Mayer-Schönberger V (2011) Delete: The virtue of forgetting in the digital age. New Jersey: Princeton University Press.

Landers RN, Brusso RC, Cavanaugh KJ and Collmus AB (2016) A primer on theorydriven web scraping: Automatic extraction of big data from the Internet for use in psychological research. Psychological methods 21(4): 475-492.

Latour B (2005) Reassembling the Social: An Introduction to Actor-Network-Theory. Oxford: Oxford University Press.

Leaver T, Highfield T, Abidin C (2020) Instagram: Visual Social Media Cultures. Cambridge, UK; Medford, MA, USA: Polity Press.

Light B, Burgess J and Duguay S (2018) The walkthrough method: An approach to the study of apps. New media \& society 20(3): 881-900.

Manovich L (2016) Instagram and contemporary image. Manovich.net. Available at: http://manovich.net/index.php/projects/instagram-and-contemporary-image (accessed 8 February 2020).

Marwick AE (2015) Instafame: Luxury selfies in the attention economy. Public culture 27(1 (75): 137-160.

Nashmi, EA and Painter DL (2018) Oh Snap: Chat Style in the 2016 US Presidential Primaries. Journal of Creative Communications 13(1): 17-33. 
Newberry C (2019) 37 Instagram Stats That Matter to Marketers in 2020. Hootsuite. Available at https://blog.hootsuite.com/instagram-statistics/ (Accessed 10 February 2020).

Page R (2013) Stories and social media: Identities and interaction. London: Routledge.

Page R (2015) The narrative dimensions of social media storytelling. In: De Fina A and Georgakopoulou A (eds.) The handbook of narrative analysis. Hoboken, US-NJ: John Wiley \& Sons, pp. 329-347.

Perriam J, Birkbak A and Freeman A (2019) Digital methods in a post-API environment. International Journal of Social Research Methodology DOI: 10.1080/13645579.2019.1682840.

Postill J and Pink S (2012) Social media ethnography: The digital researcher in a messy Web. Media International Australia 145: 123-134.

Puschmann C (2019) An end to the wild west of social media research: A response to Axel Bruns. Information, Communication \& Society, 22(11): 1582-1589.

Reidenberg JR, Breaux T, Cranor LF and French B (2015). Disagreeable Privacy Policies: Mismatches Between Meaning and Users' Understanding. Berkeley Technology Law Journal, 30(1): 39-68.

Rieder B (2015) Introducing the YouTube Data Tools. The Politics of Systems. Available at http://thepoliticsofsystems.net/2015/05/exploring-youtube/ (accessed 8 February 2020).

Rieder B, Abdulla R, Poell T, Woltering R. and Zack L (2015). Data critique and analytical opportunities for very large Facebook Pages: Lessons learned from exploring "We are all Khaled Said". Big Data \& Society 2(2): 1-22. 
Rieder B, Matamoros-Fernández A and Coromina Ò (2018) From ranking algorithms to 'ranking cultures' Investigating the modulation of visibility in YouTube search results. Convergence 24(1)50-68.

Roesner F, Gill BT and Kohno T (2014) Sex, lies, or kittens? Investigating the use of Snapchat's self-destructing messages. Proceedings of the Financial Cryptography and Data Security Conference, Christ Church, Barbados.

Rokka J and Canniford R (2016) Heterotopian selfies: how social media destabilizes brand assemblages. European Journal of Marketing 50(9/10): 1789-1813.

Rogers R (2013) Digital Methods. Cambridge, MA: MIT Press.

Rogers R (2017) Doing Web history with the Internet Archive: screencast documentaries. Internet Histories 1(1-2): 160-172.

Rogers R (2019) Doing Digital Methods. London, UK: SAGE.

Rose G (2016) Visual methodologies: An introduction to researching with visual materials. London, UK: SAGE.

Salmons JE (2015) Doing qualitative research online. London, UK: SAGE.

Senft TM and Baym NK (2015) What does the selfie say? Investigating a global phenomenon. International journal of communication 9(Feature): 1588-1606.

Venturini T and Rogers R (2019). "API-Based Research" or How can Digital Sociology and Journalism Studies Learn from the Facebook and Cambridge Analytica Data Breach. Digital Journalism 7(4): 532-540.

Von Ahn L, Blum M, Hopper NJ and Langford J (2003). CAPTCHA: Using hard AI problems for security. EUROCRYPT 2003: 297-311. 
Utz S, Muscanell N and Khalid C (2015) Snapchat elicits more jealousy than Facebook: A comparison of Snapchat and Facebook use. Cyberpsychology, Behavior, and Social Networking, 18:141-146.

Vázquez-Herrero J, Direito-Rebollal S and López-García X (2019) Ephemeral Journalism: News Distribution Through Instagram Stories. Social Media + Society 5(4): 1-13.

Warren J (2019) 10 Strategies to Drive Traffic and Sales from Instagram. Later. Available at: https://later.com/blog/drive-traffic-from-instagram/ (accessed 8 February 2020).

Waterman T (2020) Web scraping is now legal. Towards data science. Available at: https://towardsdatascience.com/web-scraping-is-now-legal-6bf0e5730a78 (accessed 8 February 2020).

Weltevrede E (2016) Repurposing Digital Methods: The Research Affordances of Platforms and Engines. Ph.D. Dissertation, Amsterdam: University of Amsterdam.

Zimmer M (2010) "But the data is already public": on the ethics of research in Facebook. Ethics and information technology 12(4): 313-325.

Zuboff S (2019) The age of surveillance capitalism: The fight for a human future at the new frontier of power. London: Profile Books. 